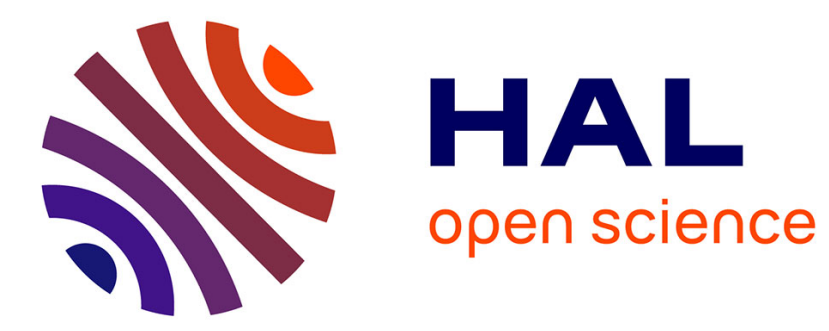

\title{
Hybrid finite volume discretization of linear elasticity models on general meshes
}

Daniele Antonio Di Pietro, Robert Eymard, Simon Lemaire, Roland Masson

\section{To cite this version:}

Daniele Antonio Di Pietro, Robert Eymard, Simon Lemaire, Roland Masson. Hybrid finite volume discretization of linear elasticity models on general meshes. Finite Volumes for Complex Applications VI, Jun 2011, Prague, Czech Republic. pp.331-339, 10.1007/978-3-642-20671-9_35 . hal-00795201

\section{HAL Id: hal-00795201 \\ https://hal.science/hal-00795201}

Submitted on 27 Feb 2013

HAL is a multi-disciplinary open access archive for the deposit and dissemination of scientific research documents, whether they are published or not. The documents may come from teaching and research institutions in France or abroad, or from public or private research centers.
L'archive ouverte pluridisciplinaire HAL, est destinée au dépôt et à la diffusion de documents scientifiques de niveau recherche, publiés ou non, émanant des établissements d'enseignement et de recherche français ou étrangers, des laboratoires publics ou privés. 


\title{
Hybrid Finite Volume Discretization of Linear Elasticity Models on General Meshes
}

\author{
Daniele A. Di Pietro, Robert Eymard, Simon Lemaire and Roland Masson
}

\begin{abstract}
This paper presents a new discretization scheme for linear elasticity models using only one degree of freedom per face corresponding to the normal component of the displacement. The scheme is based on a piecewise constant gradient construction and a discrete variational formulation for the displacement field. The tangential components of the displacement field are eliminated using a second order linear interpolation. Our main motivation is the coupling of geomechanical models and porous media flows arising in reservoir or $\mathrm{CO} 2$ storage simulations. Our scheme guarantees by construction the compatibility condition between the displacement discretization and the usual cell centered finite volume discretization of the Darcy flow model. In addition it applies on general meshes possibly non conforming such as Corner Point Geometries commonly used in reservoir and CO2 storage simulations.
\end{abstract}

Key words: hybrid finite volumes, linear elasticity, general meshes MSC2010: 74S10, 74B05

\section{Introduction}

The oil production in unconsolidated, highly compacting porous media (such as Ekofisk or Bachaquero) induces a deformation of the pore volume which (i) modifies significantly the production, and (ii) may have severe consequences such as surface subsidence or damage of well equipments. This explains the growing interest in reservoir modeling for simulations coupling the reservoir Darcy multiphase flow with the geomechanical deformation of the porous media [3]. Similarly, poromechanical models are also used in $\mathrm{CO} 2$ storage simulations to predict the over pressure induced by the injection of $\mathrm{CO} 2$ in order to assess the mechanical integrity of the storage in the injection phase.

D.A. Di Pietro, S. Lemaire, R. Masson

IFP Énergies nouvelles, FRANCE, e-mail: dipietrd, simon.lemaire, roland.masson@ifpen.fr

R. Eymard

Université Paris-Est Marne-la-Vallée, FRANCE, e-mail: robert.eymard@univ-mlv.fr 
The most commonly used geometry in reservoir and $\mathrm{CO} 2$ storage models is the so called Corner Point Geometry or CPG [7]. Although the CPG discretization is initially a structured hexahedral grid, vertical edges of the cells may typically collapse to account for the erosion of the geological layers and vertices may be dedoubled and slide along the coordlines (i.e. straight lines orthogonal to the geological layers) to model faults. In addition non conforming local grid refinement is used in near well regions. The resulting mesh is unstructured, non conforming, it includes all the degenerate cells obtained from hexahedra by collapsing edges, and hence it is not adapted to conforming finite element discretizations.

The objective of this paper is to introduce a new discretization scheme for linear elasticity equations which should

- apply on general meshes possibly non conforming;

- guarantee the stability of the coupling with Darcy flow models using cell centered finite volume discretization for the Darcy equation [1].

Our discretization is based on the family of Hybrid Finite Volume schemes introduced for diffusion problems on general meshes in [5] and also closely related to Mimetic Finite Difference schemes [6] as shown in [4]. The degrees of freedom are defined by the displacement vector $\mathbf{u}_{\sigma}$ at the center of gravity of each face $\sigma$ of the mesh as well as the displacement vector $\mathbf{u}_{K}$ at a given point $\mathbf{x}_{K}$ of each cell $K$ of the mesh. Following [5], a piecewise constant gradient is built and can be readily used to define the discrete variational formulation which mimics the continuous variational formulation for the displacement vector field. In order to stabilize this formulation and to reduce the degrees of freedom, the tangential components of the displacement are interpolated in terms of the neighbouring normal components. $\mathrm{Nu}$ merical experiments on two dimensional and three dimensional meshes show that the resulting discretization is stable and convergent. In addition, this discretization satisfies by construction the compatibility condition or LBB (see [2], [1]) condition for poroelastic models when coupled with a cell centered finite volume scheme for the Darcy flow equation.

\section{Discretization of Linear Elasticity Models}

Let $\Omega$ be a $d$-dimensional polygonal or polyhedral domain ( $d=2$ or 3 ) and let us consider the following linear elasticity problem in infinitesimal strain theory:

$$
\left\{\begin{aligned}
\operatorname{div}(\sigma(\mathbf{u}))+\mathbf{f} & =\mathbf{0} & & \text { on } \Omega, \\
\mathbf{u} & =\mathbf{u}^{D} & & \text { on } \partial \Omega^{D}, \\
\sigma(\mathbf{u}) \cdot \mathbf{n} & =\mathbf{g} & & \text { on } \partial \Omega^{N},
\end{aligned}\right.
$$

where $\mathbf{u} \in \mathbb{R}^{d}$ is the unknown displacement field and $D$ and $N$ the two exponents standing respectively for Dirichlet and Neumann boundary conditions. $\sigma(\mathbf{u})$ is the Cauchy stress tensor and is given by Hooke's law $\sigma(\mathbf{u})=2 \mu \varepsilon(\mathbf{u})+\lambda \operatorname{tr}(\varepsilon(\mathbf{u})) \mathbf{1}$, 
where $\mu, \lambda$ are the Lamé parameters and $\varepsilon(\mathbf{u})=\frac{1}{2}\left(\nabla \mathbf{u}+\nabla \mathbf{u}^{T}\right)$ is the infinitesimal strain tensor.

\subsection{Hybrid Finite Volume Discretization}

The simulation domain $\Omega$ is discretized by a set of polygonal or polyhedral control volumes $K \in \mathscr{K}$, such that $\bar{\Omega}=\bigcup_{K \in \mathscr{K}} \bar{K}$. The set of faces of the mesh is denoted by $\mathscr{E}$ and splits into boundary interfaces $\mathscr{E}_{\text {ext }}$ and inner interfaces $\mathscr{E}_{i n t}$. Among the boundary interfaces, we denote by $\mathscr{E}_{e x t}^{D}$ and $\mathscr{E}_{e x t}^{N}$ the subsets of boundary faces verifying Dirichlet or Neumann conditions. The center of gravity of the face $\sigma$ is denoted by $\mathbf{x}_{\sigma}$ and its $d-1$ dimensional measure by $|\sigma|$. A point $\mathbf{x}_{K}$ is defined inside each cell $K$ of the mesh. The set of faces of each cell $K$ is denoted by $\mathscr{E}_{K}$, and the distance between $\mathbf{x}_{K}$ and $\sigma$ by $d_{K, \sigma}$. The cone of base $\sigma \in \mathscr{E}_{K}$ and top $\mathbf{x}_{K}$ is denoted by $K_{\sigma}$.

Brief reminder of the hybrid finite volume discretization of a scalar diffusion problem (see [5]):

We first define the discrete hybrid spaces $V=\left\{\left(v_{K} \in \mathbb{R}\right)_{K \in \mathscr{K}},\left(v_{\sigma} \in \mathbb{R}\right)_{\sigma \in \mathscr{E}}\right\}$ and $V^{0}=\left\{v \in V \mid v_{\sigma}=0 \forall \sigma \in \mathscr{E}_{\text {ext }}\right\} . V^{0}$ is endowed with the discrete $H_{0, D}^{1}(\Omega)$ norm

$$
\|v\|_{V^{0}}=\left(\sum_{K \in \mathscr{K}} \sum_{\sigma \in \mathscr{E}_{K}} \frac{|\sigma|}{d_{K, \sigma}}\left|v_{\sigma}-v_{K}\right|^{2}\right)^{\frac{1}{2}} .
$$

Then, following [5], a discrete gradient is defined on each cone $K_{\sigma}$ which only depends on $v_{K}$ and $v_{\sigma^{\prime}}$ for $\sigma^{\prime} \in \mathscr{E}_{K}$. This gradient is exact on linear functions and satisfies a weak convergence property. According to [5], it can be written

$$
\nabla_{K_{\sigma}} v=\sum_{\sigma^{\prime} \in \mathscr{E}_{K}}\left(v_{\sigma^{\prime}}-v_{K}\right) \mathbf{y}_{K}^{\sigma \sigma^{\prime}} \quad \forall v \in V
$$

where $\mathbf{y}_{K}^{\sigma \sigma^{\prime}} \in \mathbb{R}^{d}$ only depends on the geometry.

Hybrid finite volume discretization of the linear elasticity model:

As we did above, we introduce $\mathbf{V}=\left\{\left(\mathbf{v}_{K} \in \mathbb{R}^{d}\right)_{K \in \mathscr{K}},\left(\mathbf{v}_{\sigma} \in \mathbb{R}^{d}\right)_{\sigma \in \mathscr{E}}\right\}$ as the discrete hybrid space. With an equivalent definition for $\mathbf{V}^{0}$, the discrete norm is now defined as $\|\mathbf{v}\|_{\mathbf{V}^{0}}^{2}=\sum_{i=1}^{d}\left\|v_{i}\right\|_{V^{0}}^{2}$. Let us also introduce the space $\mathbf{W}=\left\{\left(\mathbf{w}_{K} \in \mathbb{R}^{d}\right)_{K \in \mathscr{K}}\right.$, $\left.\left(\mathbf{w}_{\sigma} \in \mathbb{R}^{d}\right)_{\sigma \in \mathscr{E}_{\text {ext }}^{D D}},\left(w_{\sigma}^{n} \in \mathbb{R}\right)_{\sigma \in \mathscr{E}_{\text {int }} \cup \mathscr{E}_{\text {ext }}^{\text {ext }}}\right\}$ and the following projection operator $P_{\mathbf{W}}$ : $\mathbf{V} \rightarrow \mathbf{W}, \mathbf{v} \mapsto\left(\left(\mathbf{v}_{K}\right)_{K \in \mathscr{K}},\left(\mathbf{v}_{\sigma}\right)_{\sigma \in \mathscr{E}_{\text {ext }}^{D}},\left(\mathbf{v}_{\sigma} \cdot \mathbf{n}_{\sigma}\right)_{\sigma \in \mathscr{G}_{\text {int }} \cup \mathscr{E}_{\text {ext }}^{N}}\right)$, where $\mathbf{n}_{\sigma}$ is a unit vector 
normal to $\sigma$ which orientation is fixed once and for all. Let us finally define the space $\mathbf{W}^{0}=P_{\mathbf{W}}\left(\mathbf{V}^{0}\right)$ endowed with the discrete norm $\|\mathbf{w}\|_{\mathbf{W}^{0}}=\inf _{\mathbf{v} \in \mathbf{V}^{0} \mid P_{\mathbf{W}}(\mathbf{v})=\mathbf{W}}\|\mathbf{v}\|_{\mathbf{V}^{0}}$.

The main novelty of our discretization lies in the definition of a linear interpolation operator $\mathbf{I}: \mathbf{W} \rightarrow \mathbf{V}$. This linear interpolation operator must be second order accurate to preserve the order of approximation of the scheme and interpolant in the sense that $P_{\mathbf{W}}(\mathbf{I}(\mathbf{w}))=\mathbf{w}$ for all $\mathbf{w} \in \mathbf{W}$. It must also be local in the sense that it computes the displacement vector $\mathbf{v}_{\sigma}$ at a given face $\sigma \in \mathscr{E}_{\text {int }} \cup \mathscr{E}_{\text {ext }}^{N}$ in terms of a given number of normal components $\mathbf{v}_{\sigma^{\prime}} \cdot \mathbf{n}_{\sigma^{\prime}}$ taken on a stencil $\mathscr{S}_{\sigma} \subset \mathscr{E}$ of neighbouring faces $\sigma^{\prime}$ of $\sigma$ (with $\sigma \in \mathscr{S}_{\sigma}$ ). An example of construction of such an interpolator is given in subsection 2.3.

The use of the interpolation operator I will bring two crucial improvements to the discretization: first a drastic reduction of the degrees of freedom and secondly a stabilization of the discretization.

Finally, generalizing the scalar framework to the vectorial case of the linear elasticity model, we introduce a piecewise constant discrete gradient for each cone $K_{\sigma}$ :

$$
\nabla_{K_{\sigma}} \mathbf{v}=\sum_{\sigma^{\prime} \in \mathscr{E}_{K}}\left(\mathbf{v}_{\sigma^{\prime}}-\mathbf{v}_{K}\right) \otimes \mathbf{y}_{K}^{\sigma \sigma^{\prime}} \quad \forall \mathbf{v} \in \mathbf{V}
$$

\subsection{Discrete Variational Formulation}

Starting from (1), we deduce a discrete weak formulation of the problem in $\mathbf{W}^{0}$.

Setting $\varepsilon_{K_{\sigma}}(\mathbf{v})=\frac{1}{2}\left(\nabla_{K_{\sigma}} \mathbf{v}+\nabla_{K_{\sigma}} \mathbf{v}^{T}\right)$ and $\sigma_{K_{\sigma}}(\mathbf{v})=2 \mu \varepsilon_{K_{\sigma}}(\mathbf{v})+\lambda \operatorname{tr}\left(\varepsilon_{K_{\sigma}}(\mathbf{v})\right) \mathbf{1}$ for all $\mathbf{v} \in \mathbf{V}$, we introduce the discrete bilinear form on $\mathbf{W} \times \mathbf{W}$

$$
a_{\mathscr{D}}(\mathbf{u}, \mathbf{v})=\sum_{K \in \mathscr{K}} \sum_{\sigma \in \mathscr{E}_{K}}\left|K_{\sigma}\right| \sigma_{K_{\sigma}}(\mathbf{I}(\mathbf{u})): \varepsilon_{K_{\sigma}}(\mathbf{I}(\mathbf{v})) .
$$

Then, the discrete variational formulation reads: find $\mathbf{u} \in \mathbf{W}$ such that $\mathbf{u}_{\sigma}=\mathbf{u}_{\sigma}^{D}$ for all $\sigma \in \mathscr{E}$ ext and such that, for all $\mathbf{v} \in \mathbf{W}^{0}$,

$$
a_{\mathscr{D}}(\mathbf{u}, \mathbf{v})=\sum_{K \in \mathscr{K}}|K| \mathbf{f}_{K} \cdot \mathbf{v}_{K}+\sum_{\sigma \in \mathscr{E}_{\text {ext }}^{N}}|\sigma| \mathbf{g}_{\sigma} \cdot \mathbf{I}(\mathbf{v})_{\sigma}
$$

where $\mathbf{u}_{\sigma}^{D}=\frac{1}{|\sigma|} \int_{\sigma} \mathbf{u}^{D} \mathrm{~d} \sigma, \mathbf{f}_{K}=\frac{1}{|K|} \int_{K} \mathbf{f} \mathrm{d} \mathbf{x}$ and $\mathbf{g}_{\sigma}=\frac{1}{|\sigma|} \int_{\sigma} \mathbf{g} \mathrm{d} \sigma$ are average values.

It is important to keep in mind that numerical experiments show that without interpolation, the bilinear form $a_{\mathscr{D}}$ on the space $\mathbf{V} \times \mathbf{V}$ leads to an unstable scheme with vanishing eigenvalues on triangular or tetrahedral meshes with mixed Neumann Dirichlet boundary conditions.

Note also that for the solution of the linear system (6), the unknowns $\mathbf{u}_{K}$ can easily be eliminated without any fill in, reducing the degrees of freedom to the face normal components of the displacement. 


\subsection{Interpolation of the tangential components of the displacement}

Given a face $\sigma \in \mathscr{E}_{\text {int }} \cup \mathscr{E}_{\text {ext }}^{N}$, for each component $i \in \llbracket 1, d \rrbracket$ of the displacement field $\mathbf{u}_{\sigma}$, we look for a linear interpolation of the form

$$
\bar{u}_{\sigma}^{i}(\mathbf{x})=\sum_{j=1}^{d} \alpha_{\sigma}^{i j} x_{j}+\beta_{\sigma}^{i} .
$$

In order to determine the $d(d+1)$ coefficients $\left(\alpha_{\sigma}^{i j}\right)_{i, j \in \llbracket 1, d \rrbracket},\left(\beta_{\sigma}^{i}\right)_{i \in \llbracket 1, d \rrbracket}$ as linear combinations of normal components $\mathbf{u}_{\sigma^{\prime}} \cdot \mathbf{n}_{\sigma^{\prime}}, \sigma^{\prime} \in \mathscr{S}_{\sigma}$, we look for a set $\mathscr{S}_{\sigma}$ of $d(d+1)$ neighbouring faces $\sigma^{\prime}$ of the face $\sigma$, with $\sigma \in \mathscr{S}_{\sigma}$ and such that the system of equations $\overline{\mathbf{u}}_{\sigma}\left(\mathbf{x}_{\sigma^{\prime}}\right) \cdot \mathbf{n}_{\sigma^{\prime}}=\mathbf{u}_{\sigma^{\prime}} \cdot \mathbf{n}_{\sigma^{\prime}}$ is non singular. The set $\mathscr{S}_{\sigma}$ is built using the following greedy algorithm:

1. Initialization: for a given number $k>d(d+1)$, we select the $k$ closest neighbouring faces of the face $\sigma$ which are sorted from the closest to the farthest using the distance between the face center and $\mathbf{x}_{\sigma}: \sigma_{0}=\sigma, \sigma_{1}, \cdots, \sigma_{k-1}$. We set $\mathscr{S}_{\sigma}=\{\sigma\}$ and $q=1, l=0$;

2. while $q<d(d+1)$ and $l<k-1$ :

- $l=l+1$;

- if the equation $\overline{\mathbf{u}}_{\sigma}\left(\mathbf{x}_{\sigma_{l}}\right) \cdot \mathbf{n}_{\sigma_{l}}=\mathbf{u}_{\sigma_{l}} \cdot \mathbf{n}_{\sigma_{l}}$ is linearly independent with the set of equations $\overline{\mathbf{u}}_{\sigma}\left(\mathbf{x}_{\sigma^{\prime}}\right) \cdot \mathbf{n}_{\sigma^{\prime}}=\mathbf{u}_{\sigma^{\prime}} \cdot \mathbf{n}_{\sigma^{\prime}}$ for all $\sigma^{\prime} \in \mathscr{S}_{\sigma}$, then $\mathscr{S}_{\sigma}=\left\{\sigma_{l}\right\} \cup \mathscr{S}_{\sigma}$, $q=q+1$

3. if $q<d(d+1)$, the algorithm is rerun with a larger value of $k$.

Note that since $\sigma \in \mathscr{S}_{\sigma}$, the interpolation operator satisfies as required the property $P_{\mathbf{W}}(\mathbf{I}(\mathbf{u}))=\mathbf{u}$ for all $\mathbf{u} \in \mathbf{W}$.

\subsection{Compatibility condition with cellwise constant pressure for poroelastic models}

Let $L_{0}^{2}(\Omega)$ be the subspace of functions of $L^{2}(\Omega)$ with vanishing mean values. For the sake of simplicity but without any loss of generality, we consider here $\partial \Omega^{D}=\partial \Omega$ and $\mathbf{u}^{D}=\mathbf{0}$. It is well known (see [1]) that the well-posedness of linear poroelasticity models relies on the well-posedness of the following saddle point problem: find $(\mathbf{u}, p) \in H_{0}^{1}(\Omega)^{d} \times L_{0}^{2}(\Omega)$ such that

$$
\left\{\begin{aligned}
a(\mathbf{u}, \mathbf{v})+b(\mathbf{v}, p) & =(\mathbf{f}, \mathbf{v})_{L^{2}(\Omega)^{d}} & & \text { for all } \mathbf{v} \in H_{0}^{1}(\Omega)^{d} \\
b(\mathbf{u}, q) & =(h, q)_{L^{2}(\Omega)} & & \text { for all } q \in L_{0}^{2}(\Omega)
\end{aligned}\right.
$$

where $a$ is the bilinear form of the linear elasticity model and $b(\mathbf{v}, p)=-(\operatorname{div} \mathbf{v}, p)_{L^{2}(\Omega)}$. The stability of this saddle point problem results from the coercivity of $a$ and the 
following LBB condition (see [2]) which guarantees the existence and uniqueness of the solution: $\inf _{p \in L_{0}^{2}(\Omega)} \sup _{\mathbf{v} \in H_{0}^{1}(\Omega)} \frac{b(\mathbf{v}, p)}{\|\mathbf{v}\|_{H_{0}^{1}(\Omega)}\|p\|_{L^{2}(\Omega)}} \geq \gamma>0$.

The following theorem states that the LBB condition holds on the discrete spaces $\mathbf{W}^{0} \times M_{0}$, where $M_{0}$ is the space of cellwise constant functions on the mesh $\mathscr{K}$ with vanishing mean values endowed with the $L^{2}(\Omega)$ norm, and for the discrete divergence operator defined by:

$$
b_{\mathscr{D}}(\mathbf{w}, p)=-\left(\operatorname{div}_{\mathscr{D}} \mathbf{w}, p\right)_{L^{2}(\Omega)}=-\sum_{K \in \mathscr{K}} p_{K} \sum_{\sigma \in \mathscr{E}_{K} \cap \mathscr{E}_{i n t}}|\sigma| w_{\sigma}^{n}\left(\mathbf{n}_{\sigma} \cdot \mathbf{n}_{K, \sigma}\right),
$$

for all $(\mathbf{w}, p) \in \mathbf{W}^{0} \times M_{0}$, and where $\mathbf{n}_{K, \sigma}$ is the normal to the face $\sigma$ outward $K$. It implies that, assuming the coercivity of $a_{\mathscr{D}}$, the coupling of our discretization for the elasticity model with a cell centered finite volume scheme for the Darcy pressure equation will lead to a convergent and stable scheme for the poroelastic model.

Theorem 1. The bilinear form $b_{\mathscr{D}}$ defined on $\mathbf{W}^{0} \times M_{0}$ satisfies the LBB condition

$$
\inf _{p \in M_{0}} \sup _{\mathbf{w} \in \mathbf{W}^{0}} \frac{b_{\mathscr{D}}(\mathbf{w}, p)}{\|\mathbf{w}\|_{\mathbf{W}^{0}}\|p\|_{L^{2}(\Omega)}} \geq \gamma_{\mathscr{D}}>0
$$

with a constant $\gamma_{\mathscr{D}}$ depending only on usual regularity parameters of the mesh.

Proof. From the continuous LBB condition, for all $p \in M_{0}$, there exists a displacement field $\mathbf{v} \in H_{0}^{1}(\Omega)^{d}$ such that $b(\mathbf{v}, p) \geq \gamma\|\mathbf{v}\|_{H_{0}^{1}(\Omega)}\|p\|_{L^{2}(\Omega)}$. Let $\mathbf{u}$ be the element of $\mathbf{V}^{0}$ such that $\mathbf{u}_{K}=\frac{1}{|K|} \int_{K} \mathbf{v} \mathrm{d} \mathbf{x}$ for $K \in \mathscr{K}$ and $\mathbf{u}_{\sigma}=\frac{1}{|\sigma|} \int_{\sigma} \mathbf{v} \mathrm{d} \sigma$ for $\sigma \in \mathscr{E}$. Then, $\mathbf{w}=P_{\mathbf{W}}(\mathbf{u}) \in \mathbf{W}^{0}$ satisfies $b_{\mathscr{D}}(\mathbf{w}, p)=b(\mathbf{v}, p)$.

Since it is shown in [9] that $\|\mathbf{u}\|_{\mathbf{V}^{0}} \leq \kappa\|\mathbf{v}\|_{H_{0}^{1}(\Omega)}$, with a constant $\kappa$ depending on usual regularity parameters of the mesh, and since we have by definition the inequality $\|\mathbf{w}\|_{\mathbf{W}^{0}} \leq\|\mathbf{u}\|_{\mathbf{V}^{0}}$, the discrete LBB condition holds with $\gamma_{\mathscr{D}}=\frac{\gamma}{\kappa}$.

\section{Numerical experiments}

In this section, the convergence of the scheme is tested on the linear elasticity model with exact solution

$$
u_{i}=e^{\cos \left(\sum_{j=1}^{d} \chi^{i j} x_{j}\right)}, \quad i=1, \cdots, d
$$

The right hand side and the Dirichlet boundary conditions are defined by the exact solution. The Lamé parameters $\lambda$ and $\mu$ are set to 1 .

The tests have been held using an object oriented $\mathrm{C}++$ implementation which original approach is described in [8]. The relative $l^{2}$ error on the displacement and on the gradient of the displacement are plotted function of the number of inner faces. In the computation of these errors, the cellwise constant discrete solution and 
discrete gradient are used. We first consider the triangular meshes (mesh family 1), the Cartesian grids (mesh family 2), the local grid refinement (mesh family 3 ) and the Kershaw meshes (mesh family 4) from the FVCA5 2D benchmark. The exact solution is defined by

$$
\chi=\left(\begin{array}{cc}
1 & 1 \\
2 & -1
\end{array}\right) .
$$

The results presented on figure 1 show the good convergence behaviour of the scheme. The expected order of convergence is reached for all the meshes (for the solution and its gradient) and is even exceeded for the gradient on Cartesian grids. Next, let us consider the Cartesian grids (mesh family A), the randomly distorted
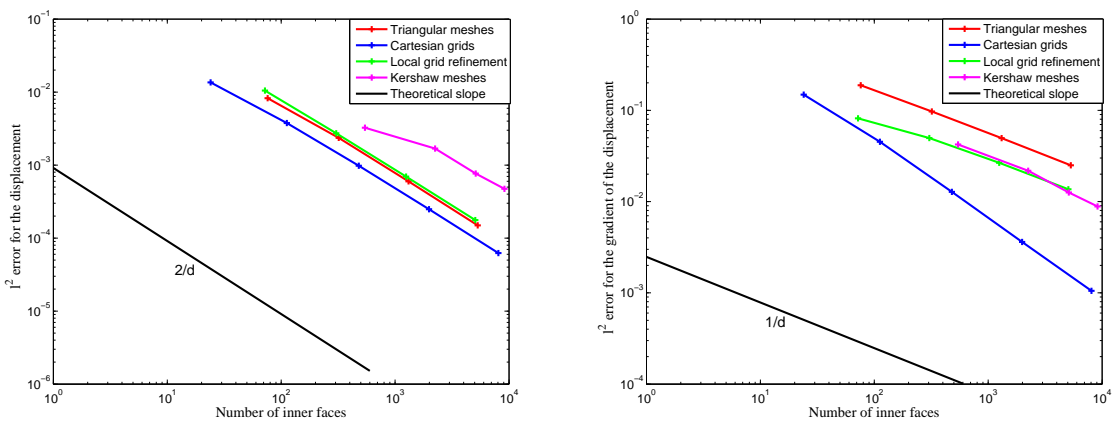

Fig. $1 l^{2}$ error for the displacement and for the gradient of the displacement function of the number of inner faces for the triangular meshes, the Cartesian grids, the local grid refinement and the Kershaw meshes.

grids (mesh family AA), and the tetrahedral meshes (mesh family B) from the FVCA6 3D benchmark. The exact solution is defined by

$$
\chi=\left(\begin{array}{ccc}
1 & 1 & 1 \\
2 & 1 & -1 \\
-1 & 1 & 2
\end{array}\right)
$$

The results exhibited on figure 2 show again the good convergence behaviour of the scheme with the expected order on the three meshes for both the discrete solution and its gradient.

\section{Conclusion}

In this paper, a new discretization method has been introduced for linear elasticity using only one degree of freedom per face. It applies to general polygonal and polyhedral meshes possibly non conforming. In addition this discretization satisfies the 

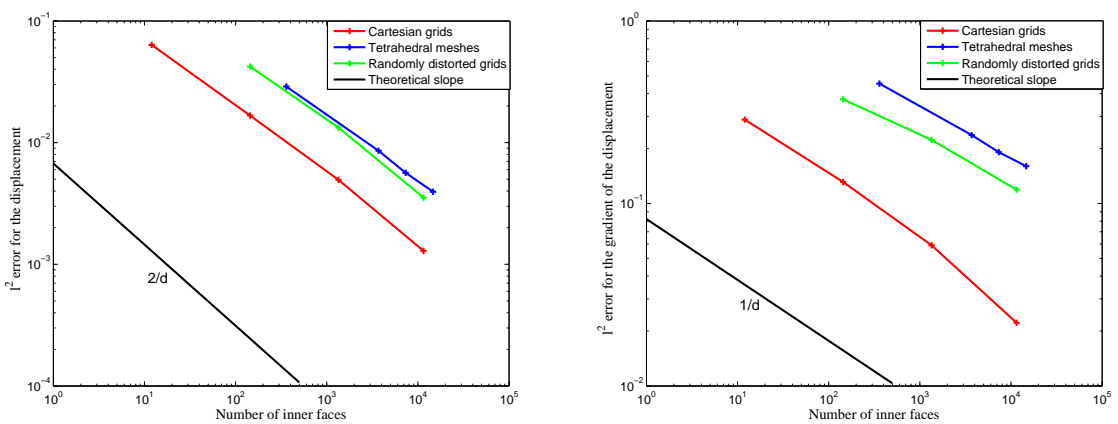

Fig. $2 l^{2}$ error for the displacement and for the gradient of the displacement function of the number of inner faces for the Cartesian grids, the randomly distorted grids and the tetrahedral meshes.

compatibility condition when coupled with cell centered finite volume schemes for the Darcy equation in poroelastic models.

First numerical experiments in 2D and 3D exhibit the stability and convergence of the scheme. In the near future, further testings will be performed on CPG grids with erosions, local grid refinement and faults, to assess the potential of this scheme for reservoir and $\mathrm{CO} 2$ storage simulations.

\section{References}

1. M.A. Murad and A.F.D. Loula. On Stability and Convergence of Finite Element Approximations of Biot's Consolidation Problem. Int. Jour. Numer. Eng., 37:645-667, 1994.

2. F. Brezzi and M. Fortin. Mixed and Hybrid Finite Element Methods. Springer-Verlag, NewYork, 1991.

3. A. Settari and F.M. Mourits. Coupling of Geomechanics and Reservoir simulation models. Comp. Meth. Adv. Geomech., Siriwardane and Zaman and Balkema, Rotterdam, 2151-2158, 1994.

4. J. Droniou, R. Eymard, T. Gallouët and R. Herbin. A unified approach to mimetic finite difference, hybrid finite volume and mixed finite volume methods. Math. Models Methods Appl. Sci., 20(2):265-295, 2010.

5. R. Eymard, T. Gallouët and R. Herbin. Discretisation of heterogeneous and anisotropic diffusion problems on general non-conforming meshes, SUSHI: a scheme using stabilisation and hybrid interfaces. IMA J. Numer. Anal., 30(4):1009-1043, 2010. See also http://hal.archivesouvertes.fr/.

6. F. Brezzi, K. Lipnikov and V. Simoncini. A family of mimetic finite difference methods on polygonal and polyhedral meshes. Math. Models Methods Appl. Sci., 15:1533-1553, 2005.

7. D.K. Ponting. Corner Point Geometry in reservoir simulation. In Clarendon Press, editor, Proc. ECMOR I, 45-65, Cambridge, 1989.

8. D.A. Di Pietro and J.M. Gratien. Lowest order methods for diffusive problems on general meshes: A unified approach to definition and implementation. These proceedings, 2011.

9. R. Eymard, T. Gallouët and R. Herbin. Finite Volume Methods. Handbook of Numerical Analysis, 7:713-1020, 2000.

The paper is in final form and no similar paper has been or is being submitted elsewhere. 